\title{
A Dosimetric Comparison of Radiotherapy Techniques in the Treatment of Carcinoma of Breast
}

\author{
Zakiya Salem Al-Rahbi, Ramamoorthy Ravichandran, Johnson Pichy Binukumar, \\ Cheriyithmanjiyil Antony Davis, Namrata Satyapal, Zahid Al-Mandhari
}

Department of Radiation Oncology, National Oncology Centre, The Royal Hospital, Muscat, Sultanate of Oman. Email: ravichandranrama@rediffmail.com

Received September $20^{\text {th }}, 2013$; revised October $18^{\text {th }}, 2013$; accepted October $26^{\text {th }}, 2013$

Copyright (C) 2013 Zakiya Salem Al-Rahbi et al. This is an open access article distributed under the Creative Commons Attribution License, which permits unrestricted use, distribution, and reproduction in any medium, provided the original work is properly cited.

\begin{abstract}
Objectives of present study are a) to compare the planning and delivery aspects of five different techniques, planned by a) forward, inverse planning and electronic tissue compensation methods; and b) to evaluate and verify the accuracy of the planning system using phantom to estimate the skin dose for target and contraletral breast from five techniques. In-vivo skin dosimetry is planned with TL detectors. Five different radiotherapy techniques for treatment of carcinoma breast were studied using archived computed tomography (CT) scans of 25 breast conserving surgery patients (leftsided whole breast), planned for $50 \mathrm{~Gy}$ in 25 fractions. Linear accelerator (Clinac $2300 \mathrm{CD}$ ) photon beams were used and thermoluminescent detectors (TLD) [LiF:Mg, Ti] estimated dose on humanoid phantom. Dose coverage (95\%) (to PTV) and hot spot (105\%) covering volumes did not show differences $(\mathrm{p}>0.05)$ in all 5 plans; Electronic compensator plans are better than others. IP-IMRT plan showed the worst Homogeneity Index $(\mathrm{HI})(\mathrm{p}<0.05)$ and needed more monitor units (MU) $(437 \pm 84)$, than other techniques. The mean doses to ipsi-lateral lung, contra-lateral breast (CB) and heart OARs ( $\mathrm{V}_{20 \text { ipsillung, }} \mathrm{CB}, \mathrm{V}_{30}$ Heart, $)$ are the least with IP-IMRT. IP-IMRT and E-COMP plans resulted in significantly lower mean dose to the superficial skin $\left(\mathrm{D}_{\text {mean }}, \mathrm{V}_{40 \text { skin, }}\right.$ 45skin, 50skin $)(\mathrm{p}<0.05)$. The mean doses estimated by TLDs were comparable or higher in 3D-CRT (D) and 3D-CRT (P) for PTV and CB; less for IP-IMRT and E-COMP compared to TPS. IP-IMRT and E-COMP techniques provide good target coverage, low doses to OARs, the least doses to the skin of PTV and contra-lateral breast and less hot spots; E-COMP showed better homogeneity, fewer MUs, and the least dose in non-target zones.
\end{abstract}

Keywords: Breast Radiotherapy; TL Detectors; Electronic Compensators; Second Malignancy

\section{Introduction}

In patients with early breast cancer, local standard therapy is breast-conserving surgery (BCS) followed by radiotherapy to the whole breast or, in the case of high-risk patients after mastectomy, radiotherapy to the chest wall with or without drainage areas [1]. The radiotherapy techniques in the treatment of breast cancer vary in different institutions, but, in general, the issue of radiation dose delivery to the chest wall after total mastectomy or to the breast following breast conservation surgery remains complex. In the conventional breast irradiation technique, the beam arrangement consists of two opposing tangential glancing portals [2], which allows acceptable coverage of the breast tissue while minimizing the dose to the adjacent critical structures (i.e., ipsilateral lung, contralateral breast, and heart). Physical or dyna- mic wedges are usually added to these tangential beams in order to compensate for the rapid changes in external contours and to improve the dose uniformity to the entire breast.

In a skin sparing intensity modulated radiotherapy (IMRT) for intact breast treatment using helical tomotherapy [3], skin was contoured as a thin strip of $5 \mathrm{~mm}$ extending from patient outline to the anterior surface of planning target volume (PTV). Plans were compared with applying dose restrictions or without, to evaluate the efficacy of them. The reduction in skin toxicity was related to the dose received by the skin PTV in the treatment of early breast cancer. Dynamic multileaf collimation (DMLC) with electronic compensation (E-COMP) softwares in planning systems has been used in some studies and compared with inverse planned IMRT and 
helical tomotherapy intensity modulation methods [4].

It was earlier reported [5] that for small field of $6 \mathrm{MV}$ $\mathrm{X}$-rays the surface doses of IMRT fields were less than open field, to the extent of $8 \%$ and $6 \%$ for $0^{\circ}$ and $75^{\circ}$ incidence. In another study, when evaluating the surface dose on the chest wall region of an anthropomorphic phantom, no significant difference was found between IMRT and conventional techniques [6]. It was also reported that the use of bolus increased the doses from 80 to $107 \%$ of the prescribed dose. The effect of bolus on the surface dose was large for chest wall tangential radiotherapy, up to an $82 \%$ increase in the presence of Aquaplast material. Yokoyama et al. [7] found that the near-surface dose (2 $\mathrm{mm}$ depth) of the IMRT field was about $10 \%$ lower than that of the open field. Other studies $[8,9]$ evaluated surface doses in tangential fields using GAF chromic films with cylindrical phantom, plane parallel chamber and MOSFET detectors.

Previously we have reported the efficacy of field in field forward planned tangential field radiotherapy in the treatment of breast cancer [10,11]. Recently it is claimed that improved homogeneity could be obtained with electronic tissue compensation [4], in which fluence distribution is optimized by the method of ray tracing, correcting for tissue deficit in each ray line. In our center, we want to implement E-COMP software in the treatment of intact breast treatments. To document the skin doses in the tangential treatments for breast, we attempted phantom study using thermo-luminescent (TL) detectors, and we compared these values in different treatment plans.

\section{Materials and Methods}

\subsection{Treatment Plans}

Treatment plans are generated in the archived computed tomography (CT) scan images of 25 breast conserving surgery patients $(<45 \mathrm{yrs})$ with left-sided whole breast, in early stage $\left(\mathrm{T}_{1-2} \mathrm{~N}_{0} \mathrm{M}_{0}\right)$ who received treatments from September 2010 to August 2012, to a dose of 50 Gy to the whole breast in 25 fractions. Left-sided breast cancer patients were selected in order to evaluate whether sparing of skin is possible while the heart is also an organat-risk. All CT scans were taken with the patient in the supine position with the arms kept above the head. The CT slices were taken at 5-mm intervals from the level of mandible to the upper abdomen.

The treatment planning system which was used for planning was Eclipse, Aria10 (version 10 A, Varian Ag, USA), grid size $2.5 \times 2.5 \mathrm{~mm}$. In the current study, five techniques were used for the treatment planning. These techniques are 3D-conformal radiotherapy 3D-CRT

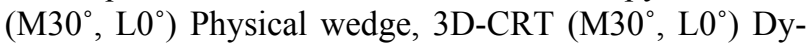
namic wedge, Field-in-Field-Forward-planned IMRT (FiF-FP-IMRT), Inverse planned IMRT (IP-IMRT) and Electronic Tissue Compensator (E-COMP). In all tech- niques, a standard tangential plan consisting of medial and lateral fields was created, according to the geometry defined during simulation.

\subsection{Target and Definition of Sensitive Structures}

The target volumes (the whole breast) and sensitive structures like heart, ipsi-lateral lung, contra-lateral lung, and contra-lateral breast, were delineated in $5 \mathrm{~mm}$ thick CT slices. The intact breast PTVs were restricted to 5 $\mathrm{mm}$ under the skin surface, to exclude the build up region from the PTVs. In the current study, the skin was countered as $4-5 \mathrm{~mm}$ strip extending from patient outline to anterior surface of PTV. Supero-inferiorly and mediolaterally, the skin extended 1 to $2 \mathrm{~cm}$ beyond the PTV (Figure 1).

The 5-mm thickness of skin was chosen to include 3 layers of skin (epidermis, dermis, and hypodermis). The field borders were clinically defined with radio-opaque wires during simulation and also delineated according to the location of the tumor, extent of breast tissue, and adequate set-up margins. The field borders were up to midline medially lower border of clavicle superiorly, and laterally and inferiorly $2 \mathrm{~cm}$ beyond the palpable breast tissue. The PTV volumes ranged from $295 \mathrm{~cm}^{3}$ to 1593 $\mathrm{cm}^{3}$, mean value $934 \pm 322 \mathrm{~cm}^{3}$.

\subsection{Comparison of Treatment Techniques}

Plans of the five different treatment techniques were compared for evaluation of dosimetric parameters. Details of the beam arrangements and objectives of plans are described below:

1) 3D-Conformal Plan, 3D-CRT (P):

In this conventional planning technique, the beam arrangement consisted of two parallel opposing tangential beams ensuring the best possible coverage of the breast tissue and minimizing the dose to the adjacent critical structures (i.e., ipsilateral lung, contraletral breast, and heart). The "isocentre" of the treatment machine is positioned at centre point of the midline joining two parallel opposing fields. $30^{\circ}$ Physical wedges were then added to medial tangential beam in order to improve the dose uniformity to the PTV, and to compensate for the rapid

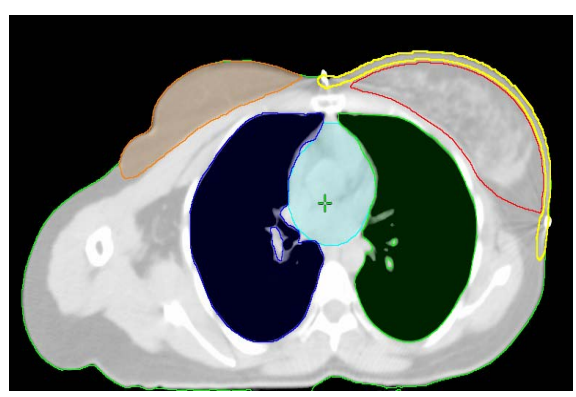

Figure 1. PTV, OARs and skin delineation. 
changes in external contours. Efforts were made to minimize volumes of heart and lung which unavoidably get included within the field borders.

2) 3D-Conformal Plan, 3D-CRT (D):

This technique is same like the previous plan, but $30^{\circ}$ dynamic wedge is replaced instead of physical wedge in the medial tangential beam.

3) Field-in-Field-Forward-planned IMRT (FiF-FPIMRT):

Two open tangential fields were created in this technique, according to the geometry defined during simulation to achieve uniform dose distribution to the breast volume (adequate coverage to the tumor bed), limiting the volume of the heart receiving a dose $>20$ Gy not to exceed $5 \%$, minimizing hot spot regions as well as limiting dose to the ipsilateral lung and contra-lateral breast. The "isocentre" of the treatment machine is positioned at same point as for 3D-conformal plan. Initially, equal weights were assigned to the two open fields, and the corresponding dose distribution was calculated. By viewing the $95 \%$ dose cloud in a beam's eye view projection of the treatment fields, subfields were manually designed to boost the area not included in the dose cloud. The shape of each subfield was iteratively modified (by forward plan) with aided visualization of $105 \%$ dose clouds in the beam's eye view. The number of subfields varied from 3 to 4 . Finally, the main field and subfields were merged in one portal, including several MLC segments for sequential irradiation [10].

4) Inverse planned IMRT (IP-IMRT):

IP-IMRT optimized plans were generated to achieve the same objectives described for FiF-FP-IMRT plan. A tangential IMRT plan consisting of medial and lateral open fields like the ones in the standard tangential plan. In these 2 fields a few IMRT segments gets added to achieve optimization. The "isocentre" of the treatment machine is positioned at the same point as in 3D-CRT and FiF-FP-IMRT plans. After optimization was done, fluence maps were extended beyond the patient surface to provide skin flash and were converted to leaf sequences for DMLC delivery.

5) Electronic Tissue Compensation (E-COMP):

E-COMP is an approach to intensity modulation that seeks to improve dose homogeneity within a target by using the leaves of a multileaf collimator, in dynamic mode, to compensate for variations in the target shape and density. Like the IP-IMRT, Fluence maps were extended beyond the patient surface to provide skin flash and were converted to leaf sequences for DMLC delivery.

\subsection{Evaluation of Plans}

The generated treatment plans were compared objectively, using the dose volume histograms (DVH) for
PTVs and different Organs at Risk (OAR) regions of interest. For comparing each parameter, the statistical significance was calculated by $\mathrm{p}$-value analysis from student's t-test. $\mathrm{V}_{95}, \mathrm{D}_{\text {mean, }}, \mathrm{D}_{\max }$, Homogeneity index (HI) were compared for all these five techniques. For heart OAR the values of $V_{\text {mean }}, V_{30}$; for ipsi-lateral lung $V_{\text {mean }}$, $\mathrm{V}_{20}$ dose value; for contra-lateral breast $\mathrm{V}_{\text {mean }}$, dose value and $\mathrm{V}_{40}, \mathrm{~V}_{45}, \mathrm{~V}_{50}$ doses for skin were evaluated and compared with dose to PTV.

The following parameters were used for objective evaluation of the plans:

1) Relative volume of breast PTV receiving $105 \%$ of the prescription dose $\left(\mathrm{V}_{105 \%}\right)$ (Represent the extension of hot-spot regions within the breast).

2) Mean $\left(D_{\text {mean }}\right)$ and Maximum dose $\left(D_{\max }\right)$ delivered to the target volume.

3) Target volume receiving $95 \%$ of the dose, $\left(\mathrm{V}_{95 \%}\right)$.

4) Homogeneity index (HI) in PTV defined by the relation

$$
\mathrm{HI}=\left(\mathrm{D}_{\text {maxm }}-\mathrm{D}_{\text {minm }}\right) / \mathrm{D}_{\text {mean }}
$$

5) Relative volume of a given tissue receiving $20 \mathrm{~Gy}$ or $30 \mathrm{~Gy}$ and the mean doses, $\mathrm{V}_{20 \mathrm{~Gy}}$ for the ipsi-lateral lung and the $\mathrm{V}_{30 \mathrm{~Gy}}$ for the heart.

6) The mean dose of heart, ipsi-lateral lung and contraletral breast.

7) Relative volume of the skin receiving dose (40, 45, $50 \mathrm{~Gy})$ and the mean dose.

\subsection{In Vivo Skin Dosimetry Study}

This work aimed to achieve two purposes, 1) to verify the accuracy of the planning system to calculate the skin dose for PTV and contraletral breast 2) to evaluate the magnitude of difference between the plans. Anthropomorphic phantom (Rando, Alderson Radiation Therapy, Long Beach, British Columbia, Canada) with an attached breast was used. This phantom was scanned by CT with $5 \mathrm{~mm}$ cut thickness. Then, CT cuts were transferred to treatment planning system (ARIA ver. 10, Varian, Milpitas, USA) where the PTV and OARs were delineated. Same plans previously applied for patients in each technique, were carried out for the phantom PTV. Phantom is irradiated for $500 \mathrm{cGy}$ to PTV for 3 fractions.

\subsection{Skin Dose Estimates}

\subsubsection{Calibration of TL Detectors}

TLD chips of $3.2 \mathrm{~mm} \times 3.2 \mathrm{~mm} \times 0.9 \mathrm{~mm}$ size $\mathrm{LiF}: \mathrm{Mg}$, Ti (Bicron Harshaw, Germany) were calibrated after annealing in oven standard annealing treatment $\left(400^{\circ} \mathrm{C}\right.$ for $1 \mathrm{~h}$ followed fast cool on removal from oven and then at $80^{\circ} \mathrm{C}$ for $24 \mathrm{~h}$ ). $72 \mathrm{TLD}$ chips in plastic plate were irradiated to a dose of $200 \mathrm{MU}$ at $1.5 \mathrm{~cm}$ depth in the perpex phantom, with a field size of $10 \times 10 \mathrm{~cm}^{2}$ at $100 \mathrm{~cm}$ SSD in Clinac 2300 CD linear accelerator. These TLDs were 
read by TLD reader (5500 Model, Bicron Harshaw, Germany) and VinRFMs software. The TLDs which had same response where chosen and then 2 TLD chips were packed together in small plastic bag. Two sets of TLDs were selected for high and low dose calibration, each set irradiated to 199MU (2 Gy) and 3 MU (3 cGy).

\subsubsection{Irradiation}

The five TLD packs were placed on the skin of anthropomorphic phantom at superior, inferior, medial, lateral, and central locations of irradiated breast, and another five kept in corresponding points of contra-lateral breast (Refer Figure 2). The phantom was placed on the treatment table, and all 5 plans were executed in Clinac $2300 \mathrm{CD}$ linear accelerator same positions, three irradiations were given for each plan respectively, to an irradiation of 500 MU for each field. A total of 30 measurements for PTV breast and contra-lateral breast were made available for dose estimates. Also the overall mean skin dose and average dose difference was taken at each of these positions, as well as the overall average to assess the mean difference in skin dose.

\section{Results}

\subsection{Dosimetric Parameters}

Table 1 shows the comparison of average dosimetric

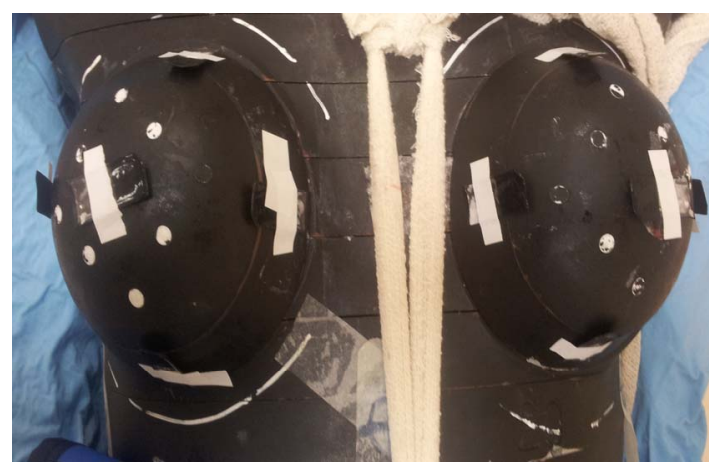

(a)

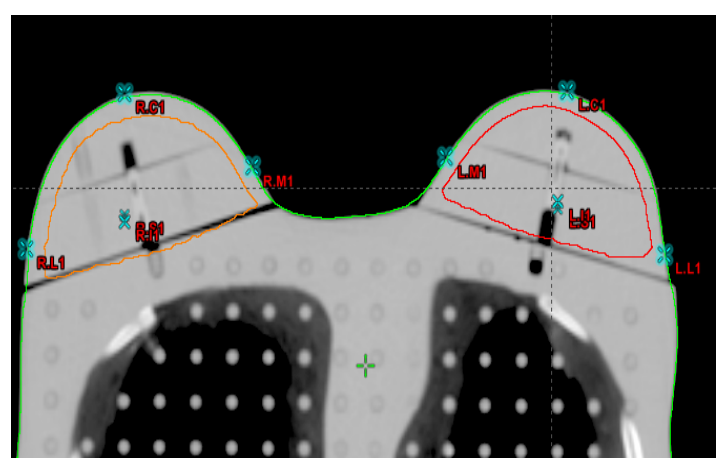

(b)

Figure 2. (a) Locations TLDs on the skin in phantom; (b) CT scan at mid plane showing both breasts. characteristics for PTV. It is observed that all the 5 plans, 3D-CRT(P), 3D-CRT(D), FiF-FP-IMRT, IP-IMRT and E-COMP achieved comparable good dose coverage, delivering prescribed dose more than $95 \%$ to $>95 \%$ of the breast PTV; with $105 \%$ of dose (hot regions) was observed in less than $10 \%$ of the target volume. $\mathrm{D}_{\max }$. dose was less than $110 \%$ of the prescribed dose in all plans except for IP-IMRT $\left(\mathrm{D}_{\text {max. }}=120.12 \pm 11.42\right)$ than 3D-CRT (D) plan $\left(\mathrm{D}_{\text {max. }}=111 \pm 2.4\right)$. ECOMP plan resulted in a smaller hot spot within the breast volume $(3.7 \%)$ than the other plans, whereas 3D-CRT (D) plan had larger hotspot area $(8.44 \%)$ within the PTV than the other plans. The differences between the plans were not significant $(\mathrm{p}>$ $0.05)$.

All plans had good homogeneity inside the PTV (refer last row Table 1), expect for IP-IMRT which had higher HI value. The difference between the IP-IMRT plan and the other plans were statistically significant $(\mathrm{p}<0.05)$.

Table 2 shows the average Monitor Units (MU) required to deliver for all plans. The average MUs needed to deliver IP-IMRT plan $(437 \pm 84)$ was about twice the value needed to deliver 3D-CRT (P), 3D-CRT (D) and FiF-FP-IMRT. The comparison of average dosimetric characteristics for Organ At Risks (OARs) is presented in Table 3. It can be seen that, all the values are less than the limits of tolerance. Also all plans are equivalent in sparing critical organs. Although the differences between planning techniques do not show statistical significance, a small decrease in the dose to OARs was present in the IP-IMRT and E-COMP plans. Table 4 shows the dose volume data for skin OAR. In terms of $\mathrm{V}_{40 \mathrm{~Gy}}$ and $\mathrm{V}_{45 \mathrm{~Gy}}$, $\mathrm{V}_{50 \text { Gy }}$ IP-IMRT and E-COMP treatment plans have an edge over 3D-CRT (P), 3D-CRT (D) and FiF-FP-IMRT plans. Maximum dose received is higher for IP-IMRT plan vis-a-vis other plans. Figure 3 shows the DVH patterns of skin for all the 5 different treatment plans, from which the above dosimetric parameters were derived. It could be observed from Figure 3 that E-COMP and IP-IMRT plans give lowest $\mathrm{V}_{40 \mathrm{~Gy}}, \mathrm{~V}_{45 \mathrm{~Gy}}, \mathrm{~V}_{50 \mathrm{~Gy}}$ compared to other 3 plans. Also IP-IMRT plans have maximum dose value going up to $122 \%$.

\subsection{TLD Skin Dose Estimates}

Tables 5 and $\mathbf{6}$ show the doses estimated in all the five treatment plans in the anthropomorphic phantom for PTV breast and contralateral breast skin. The calculated and measured doses were comparable within for 3DCRT-D, 3DCRT-P treatments. However, there were discrepancies at some points in the case of ETC and IP-IMRT plans. For FIF-IMRT plans variations up to $\pm 20 \%$ for 3 out of 5 locations. TPS dose estimates are lower in all the points in target breast, and the measurements showed always higher doses in both IP-IMRT and E-COMP plans. Superior part of breast showed less dose values in TLD meas- 
Table 1. Comparison of average dosimetric characteristics for PTV.

\begin{tabular}{cccccc}
\hline Dosimetric Parameter & CRT $(\mathrm{P})$ & CRT $(\mathrm{D})$ & FiF-IMRT & IP-IMRT & E-COMP \\
\hline $\mathrm{V}_{95}$ & $96.4 \pm 1.3$ & $96.4 \pm 1.2$ & $96.3 \pm 1.2$ & $96.2 \pm 1.0$ & $96.0 \pm 1.1$ \\
$\mathrm{~V}_{105}$ & $6.9 \pm 4.6$ & $8.4 \pm 4.5$ & $4.1 \pm 4.7$ & $4.6 \pm 3.9$ & $3.7 \pm 5.5$ \\
$\mathrm{D}_{\text {mean }}$ & $100.5 \pm 0.5$ & $100.5 \pm 0.6$ & $100.3 \pm 0.7$ & $101.0 \pm 0.7$ & $100.5 \pm 0.7$ \\
$\mathrm{D}_{\text {maxm }}$ & $109.6 \pm 1.6$ & $111.0 \pm 2.4$ & $107.6 \pm 1.9$ & $120.1 \pm 11.4$ & $109.7 \pm 5.0$ \\
$\mathrm{D}_{\text {minm }}$ & $77.2 \pm 9.2$ & $79.6 \pm 9.4$ & $79.8 \pm 9.5$ & $68.5 \pm 6.7$ & $72.4 \pm 9.5$ \\
$\mathrm{HI}$ & $\mathbf{0 . 3 2} \pm \mathbf{0 . 1}$ & $\mathbf{0 . 3 4} \pm \mathbf{0 . 1 0}$ & $\mathbf{0 . 3 1} \pm \mathbf{0 . 1}$ & $\mathbf{0 . 5 1} \pm \mathbf{0 . 2}$ ("significant) $^{*}$ & $\mathbf{0 . 3 7} \pm \mathbf{0 . 1}$ \\
\hline
\end{tabular}

Table 2. Monitor Units (MU) (Mean) to execute different treatment plans.

\begin{tabular}{cccccc}
\hline Compared Parameter & CRT $($ P) & CRT (D) & FiF-IMRT & IP-IMRT & ECOMP \\
\hline Med. Tang & $168 \pm 10$ & $124 \pm 6$ & $114 \pm 5$ & $206 \pm 37$ & $170 \pm 47$ \\
Latr. Tang & $111 \pm 15$ & $107 \pm 7$ & $114 \pm 5$ & $238 \pm 66$ & $151 \pm 42$ \\
Total MU & $\mathbf{2 7 8} \pm \mathbf{1 5}$ & $\mathbf{2 3 5} \pm \mathbf{1 0}$ & $\mathbf{2 2 7} \pm \mathbf{9}$ & $\mathbf{4 3 7} \pm \mathbf{8 4}$ & $\mathbf{3 0 6} \pm \mathbf{8 4}$ \\
\hline
\end{tabular}

Table 3. Dose-volume analysis for organs at risk (OARs).

\begin{tabular}{|c|c|c|c|c|c|}
\hline OAR Dose \& Volume & CRT (P) & CRT (D) & FiF-IMRT & IP-IMRT & E COMP \\
\hline Heart $D_{\text {mean }}$ & 6.7 & 5.9 & 6.1 & 5.1 & 6.0 \\
\hline $\mathrm{D}_{\operatorname{maxm}}$ & 9.1 & 87.7 & 87.4 & 88.3 & 86.2 \\
\hline $\mathrm{D}_{\text {minm }}$ & 1.2 & 1.1 & 1.2 & 1.0 & 1.1 \\
\hline $\mathrm{V}_{30 \text { Gy }}$ & 2.3 & 2.2 & 2.6 & 1.5 & 2.0 \\
\hline Lung $\mathrm{D}_{\text {mean }}$ & 11.6 & 11.2 & 11.3 & 9.6 & 10.6 \\
\hline $\mathrm{D}_{\operatorname{maxm}}$ & 97.1 & 100.8 & 98.4 & 100.5 & 99.5 \\
\hline $\mathrm{D}_{\text {minm }}$ & 0.6 & 0.6 & 0.5 & 0.5 & 0.6 \\
\hline $\mathrm{V}_{20 \mathrm{~Gy}}$ & 8.6 & 8.4 & 8.7 & 6.7 & 7.9 \\
\hline C. Lat. $\mathrm{D}_{\text {mean }}$ & 1.3 & 1.2 & 1.1 & 1.0 & 1.2 \\
\hline Breast $D_{\operatorname{maxm}}$ & 25.9 & 24.8 & 29.3 & 21.7 & 22.0 \\
\hline $\mathrm{D}_{\text {minm }}$ & 0.1 & 0.1 & 0.1 & 0.1 & 0.1 \\
\hline
\end{tabular}

Table 4. Dose-volume analysis for skin OAR.

\begin{tabular}{cccccc}
\hline OAR, Dose \& Volume & CRT (P) & CRT (D) & FiF-IMRT & IP-IMRT & E COMP \\
\hline SkinD $D_{\text {mean }}$ & 70.9 & 71.9 & 71.3 & 64.0 & 68.0 \\
$\mathrm{D}_{\text {maxm }}$ & 108.0 & 109.0 & 106.0 & 122.0 & 106.0 \\
$\mathrm{D}_{\text {minm }}$ & 0.5 & 0.4 & 0.5 & 0.4 & 0.4 \\
$\mathrm{~V}_{40}$ Gy & 52.5 & 52.8 & 56.9 & 39.7 & 45.9 \\
$\mathrm{~V}_{45 \text { Gy }}$ & 24.9 & 26.9 & 25.1 & 14.7 & 15.9 \\
$\mathrm{~V}_{50}$ & 6.5 & 5.3 & 4.1 & 3.4 & 2.8 \\
\hline
\end{tabular}

urements, and when the experiments were repeated they confirmed same results.

In Table $\mathbf{6}$ it could be observed that the measured dose was always higher than calculated dose for the contra lateral breast. For contralateral breast, centre of the field (nipple level) and the medial part of the breast are at a higher dose level compared to other 3 locations around the breast. Dose at contralateral breast expressed as percentage of doses (both for TLD measured and TPS calculated) at corresponding points of PTV breast is shown in Table 7. 3D-CRT(P) has shown higher percentage of PTV dose compared to 3D-CRT(D) but the difference is 


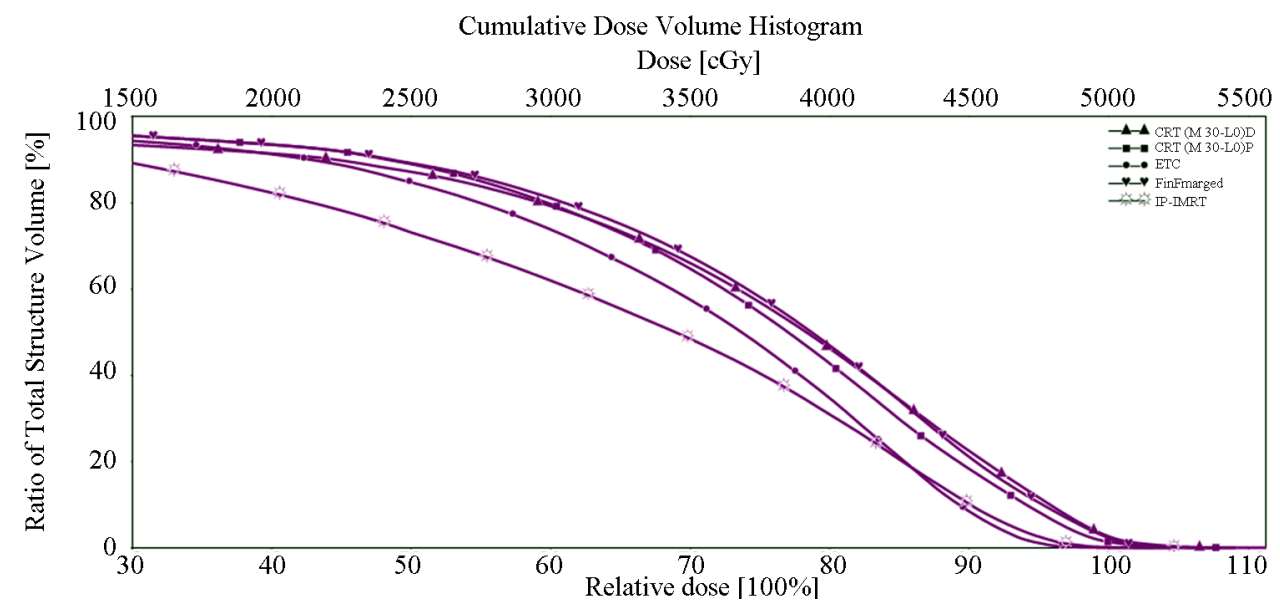

Figure 3. Comparison of dose volume histograms (DVHs) for skin OAR for all treatment plans.

Table 5. TLD measured and TPS calculated doses (cGy) in breast PTV.

\begin{tabular}{ccccccccccc}
\hline & \multicolumn{2}{c}{ CRT (P) } & \multicolumn{2}{c}{ CRT (D) } & \multicolumn{2}{c}{ FiF-IMRT } & \multicolumn{2}{c}{ IP-IMRT } & \multicolumn{2}{c}{ ECOMP } \\
\cline { 2 - 10 } Location & \multicolumn{2}{c}{ Dose estimates } & \multicolumn{2}{c}{ Dose estimates } & \multicolumn{2}{c}{ Dose estimates } & \multicolumn{2}{c}{ Dose estimates } & \multicolumn{2}{c}{ Dose estimates } \\
\cline { 2 - 11 } & TLD & TPS & TLD & TPS & TLD & TPS & TLD & TPS & TLD & TPS \\
\hline Centre & 464 & 455.3 & 508 & 467.9 & 447 & 476.5 & 507 & 198.7 & 249 & 206.5 \\
Superior & 390 & 504.3 & 505 & 494.7 & 164 & 496.4 & 198 & 196.0 & 150 & 108.5 \\
Inferior & 543 & 481.8 & 547 & 504.8 & 473 & 471.4 & 451 & 115.7 & 476 & 184.6 \\
Lateral & 441 & 459.4 & 431 & 454.2 & 387 & 455.6 & 375 & 277.1 & 394 & 280.4 \\
Medial & 359 & 394.9 & 380 & 424.5 & 339 & 413.0 & 578 & 247.1 & 593 & 249.1 \\
\hline
\end{tabular}

Table 6. TLD measured doses (cGy) in contralateral breast.

\begin{tabular}{|c|c|c|c|c|c|c|c|c|c|c|}
\hline \multirow{3}{*}{ Location } & \multirow{2}{*}{\multicolumn{2}{|c|}{$\frac{\text { CRT }(\mathrm{P})}{\text { Dose estimates }}$}} & \multirow{2}{*}{\multicolumn{2}{|c|}{$\frac{\text { CRT (D) }}{\text { Dose estimates }}$}} & \multirow{2}{*}{\multicolumn{2}{|c|}{$\frac{\text { FiF-IMRT }}{\text { Dose estimates }}$}} & \multirow{2}{*}{\multicolumn{2}{|c|}{$\frac{\text { IP-IMRT }}{\text { Dose estimates }}$}} & \multicolumn{2}{|c|}{ ECOMP } \\
\hline & & & & & & & & & Dos & nates \\
\hline & TLD & TPS & TLD & TPS & TLD & TPS & TLD & TPS & TLD & TPS \\
\hline Centre & 22.5 & 6.6 & 18.1 & 6.4 & 6.0 & 6.6 & 12.0 & 5.5 & 10.5 & 5.7 \\
\hline Superior & 9.30 & 2.1 & 6.70 & 2.0 & 3.0 & 2.1 & 4.6 & 1.8 & 3.1 & 1.8 \\
\hline Inferior & 12.7 & 1.8 & 10.1 & 1.8 & 4.0 & 3.8 & 16.6 & 1.8 & 15.1 & 1.5 \\
\hline Lateral & 11.2 & 1.7 & 7.20 & 1.6 & 2.5 & 1.7 & 4.0 & 1.4 & 3.4 & 1.4 \\
\hline Medial & 27.1 & 8.8 & 23.7 & 7.8 & 11.0 & 7.8 & 7.1 & 6.8 & 17.1 & 6.9 \\
\hline
\end{tabular}

about $1.0 \%$ at all 5 locations of the contralateral breast.

\section{Discussion}

The present report estimated the various dosimetric parameters compared in the different treatment techniques generally applied in radiotherapy, used as adjuvant to breast conserving surgery. Tolerance of skin and cosmesis in breast mainly depends on the delivered radiation doses. Therefore, it is necessary to document these dose values for various techniques. As treatment planning softwares may not address the exact dose delivery, especially in oblique incidence of beams, as well as scattered radiation and corpuscular electron patterns, it is felt that there is need to estimate the doses by measurements.
Comparison of five different treatment plans in terms of evaluated dose pattern showed that IP-IMRT and ECOMP plans resulted in a significant skin dose reduction without either compromising the coverage or dose homogeneity of the clinical target (i.e., breast) or increasing the dose to other organs-at-risk. The most important aspect of our study is the reduction in skin dose (both $V_{40}$ Gy and $\mathrm{V}_{45 \text { Gy }}$ of skin) by configuring the skin as an OAR. For the patients with early breast cancer, the cosmetic outcome depends on the treatment plans in adjuvant radiotherapy, and therefore the above results assume importance. Some studies have shown that, the areas of large dose inhomogeneities $(>10 \%)$ may be related to significant radiation-induced, acute skin toxicity, including 
Table 7. Doses (cGy) to contralateral breast as \% dose of PTV.

\begin{tabular}{ccccccccccc}
\hline & \multicolumn{2}{c}{ CRT $(\mathrm{P})$} & \multicolumn{2}{c}{ CRT $(\mathrm{D})$} & \multicolumn{2}{c}{ FiF-IMRT } & \multicolumn{2}{c}{ IP-IMRT } & \multicolumn{2}{c}{ ECOMP } \\
\cline { 2 - 11 } Location & \multicolumn{2}{c}{ Dose estimates } & \multicolumn{2}{c}{ Dose estimates } & \multicolumn{2}{c}{ Dose estimates } & \multicolumn{2}{c}{ Dose estimates } & \multicolumn{2}{c}{ Dose estimates } \\
\cline { 2 - 12 } & TLD & TPS & T LD & TPS & TLD & TPS & TLD & TPS & TLD & TPS \\
\hline Centre & 4.90 & 1.45 & 3.56 & 1.37 & 1.34 & 1.38 & 2.30 & 2.80 & 4.22 & 2.76 \\
Superior & 2.14 & 0.42 & 1.33 & 0.40 & 1.64 & 0.42 & 2.32 & 0.51 & 2.07 & 1.65 \\
Inferior & 2.33 & 0.38 & 1.85 & 0.36 & 0.85 & 0.38 & 3.68 & 1.56 & 3.17 & 0.76 \\
Lateral & 2.54 & 0.37 & 1.67 & 0.35 & 0.65 & 0.38 & 1.07 & 0.51 & 0.86 & 0.49 \\
Medial & 7.50 & 2.23 & 6.24 & 1.89 & 3.24 & 1.89 & 1.23 & 2.75 & 2.89 & 2.89 \\
\hline
\end{tabular}

the presence of breast erythema with patchy desquamation and pitting edema. The IP-IMRT plan had the highest maximum dose, it was $(120 \pm 11.4)$ of the prescribed dose, but has less PTV volume that received $105 \%$ of the prescribed dose.

Present TLD measured values in phantom using the various treatment plans confirmed significant differences, which are consistent with earlier studies [8,9]. By contouring part of the skin volume, and earmarking as "skin OAR" volume receiving 40 Gy to 50 Gy is significantly reduced by about $20 \%$, though there was no compromise on coverage of PTV [3]. It was reported earlier in some studies [12] that the beam obliquity has a large effect on the surface dose. The highest surface dose has been observed where the beam is more tangential to the surface. The effect of breast size on scatter dose to contralateral breast has also been studied earlier [13]. In their study, 65 patients of breast cancer using $6 \mathrm{MV}$ photon with IMRT technique measured contralateral breast dose using TLD. The primary breast size volume was calculated by planning system from CT slices. They found the mean contralateral dose of $7.2 \%$ of primary breast dose (5000 cGy) and found that the contribution to contralateral breast dose is strongly dependent on primary breast size of the patient. Therefore it became of more concern in young breast cancer patients with bulky protuberant breast. In the above context, our present study showing dose in the range of 2 to $7 \%$ is in agreement.

\section{Conclusion}

In our dosimetric comparison of five techniques in 25 patients with left-sided early-stage breast cancer $\&$ phantom study, IP-IMRT \& E-COMP techniques provided good target coverage and maintained low doses to OARs and fewer doses to the skin of PTV and contraletral breast. In addition, these two techniques provided a smaller hot spot within the breast volume, which may correlate with improved cosmetic outcomes. E-COMP technique had better homogeneity inside PTV than IP-IMRT and also fewer MUs than IP-IMRT, reducing total radiation exposure, avoided low dose spill of radiation to structures at risk for second malignancy. In addition, IP-
IMRT may not be preferred because of the motion of the chest wall during treatment due to normal breathing.

\section{Acknowledgements}

Authors thank Director General, Royal Hospital for kind permission obtained for the study.

\section{REFERENCES}

[1] U. Veronesi, E. Marubini, L. Marian, V. Galimberti, A. Luini, P. Veronesi, et al., "Radiotherapy after Breast Conserving Surgery in Breast Cancer: Long Term Result of Randomized Trial," Annals of Oncology, Vol. 12, No. 7, 2001, pp. 997-1003.

http://dx.doi.org/10.1023/A:1011136326943

[2] A. Recht, "Breast Cancer: Stages T1 and T2," In: L. L. Gunderson and J. E. Tepper, Eds., 2nd Edition, Clinical Radiation Oncology, Churchill Livingstone, Elsevier Publications, 2007, pp. 1475-1495.

[3] E. P. Saibishkumar, M. A. Mackenzie, D. Severin, A. Mihai, J. Hanson, H. Daly, G. Fallone, M. B. Parliament and B. Abdulkarim, "Skin-Sparing Radiation Using Intensity-Modulated Radiotherapy after Conservative Surgery in Early-Stage Breast Cancer: A Planning Study," International Journal of Radiation Oncology Biological Physics, Vol. 70, No. 2, 2008, pp. 485-491. http://dx.doi.org/10.1016/j.ijrobp.2007.06.049

[4] J. Caudell, F. Jennifer, D. L. Santos, K. S. Keene, J. B. Fiveash, W. Wang and J. D. Carlisle, "A Dosimetric Comparison of Electronic Compensation, Conventional Intensity Modulated Radiotherapy, and Tomotherapy in $\mathrm{Pa}$ tients with Early-Stage Carcinoma of the Left Breast Jimmy Popple," International Journal of Radiation Oncology Biological Physics, Vol. 68, No. 5, 2007, pp. 1505-1511. http://dx.doi.org/10.1016/j.ijrobp.2007.04.026

[5] N. Dogan and G. P. Glasgow, "Surface and Build-Up Region Dosimetry for Obliquely Incident Intensity Modulated Radiotherapy 6 MV X-Rays," Medical Physics, Vol. 30, No. 12, 2003, pp. 3091-3096. http://dx.doi.org/10.1118/1.1625116

[6] S. H. Hsu, P. L. Roberson, Y. Chen, R. B. Marsh, L. J. Pierce and J. M. Moran, "Assessment of Skin Dose for Breast Chest Wall Radiotherapy as a Function of Bolus Material," Physics in Medicine and Biology, Vol. 53, No. 10, 2008, pp. 2593-2606. 
http://dx.doi.org/10.1088/0031-9155/53/10/010

[7] S. Yokoyama, P. L. Roberson, D. W. Litzenberg, J. M. Moran and B. A. Fraass, "Surface Buildup Dose Dependence on Photon Field Delivery Technique for IMRT," Journal of Applied Clinical Medical Physics, Vol. 5, No. 2, 2004, pp. 71-81. http://dx.doi.org/10.1120/jacmp.2020.21706

[8] M. Nakano, R. F. Hill, M. Whitaker, J. H. Kim and Z. J. Kuncic, "A Study of Surface Dosimetry for Breast Cancer Radiotherapy Treatments Using Gafchromic EBT2 Film," Journal of Applied Clinical Medical Physics, Vol. 13, No. 3, 2012, pp. 83-96.

[9] K. Y. Quach, J. Morales, M. J. Butson, A. B. Rosenfeld and P. E. Metcalfe, "Measurement of Radiotherapy X-Ray Skin Dose on a Chest Wall Phantom," Medical Physics, Vol. 27, No. 7, 2000, pp. 1676-1680.

[10] K. Krishnamurthy, S. S. Sivakumar, C. A. Davis, R. Ravichandran and K. ElGhamrawy, "Optimization of Dose Distribution with Multi-Leaf Collimator Using Field-inField Technique for Parallel Opposing Tangential Beams of Breast Cancers," Journal of Medical Physics, Vol. 33, No. 2, 2008, pp. 60-63. http://dx.doi.org/10.4103/0971-6203.41194
[11] Z. S. Al-Rahbi, Z. Al Mandhari, R. Ravichandran, F. Al-Kindi, C. A. Davis, S. Bhasi, N. Satyapal and B. Rajan, "Dosimetric Comparison of Intensity Modulated Radiotherapy Isocentric Field Plans and Field in Field forward Plans in the Treatment of Breast Cancer," Journal of Medical Physics, Vol. 38, No. 1, 2013, pp. 34-41. http://dx.doi.org/10.4103/0971-6203.106601

[12] A. Kelly, N. Hardcastle, Metcalfe, D. Cutajar, A. Quinn, K. Foo, M. Cardoso, S. Barlin and A. Rosenfeld, "Surface Dosimetry for Breast Radiotherapy in the Presence of Immobilization Cast Material," Physics in Medicine and Biology, Vol. 56, No. 4, 2011, pp. 1001-1013. http://dx.doi.org/10.1088/0031-9155/56/4/008

[13] A. K. Bhatnagar, D. E. Heron, M. Deutch, E. Brandner, W. U. Andrew and S. Kalnicki, "Does Breast Size Affect the Scatter Dose to Ipsilateral Lung, Heart or Contralateral Breast in Primary Irradiation Using Intensity Modulated Radiotherapy (IMRT)," American Journal of Clinical Oncology, Vol. 29, No. 1, 2006, pp. 80-84. http://dx.doi.org/10.1097/01.coc.0000198743.80991.15 